\title{
Análisis biomecánico del efecto de un aumento del ángulo de salida en el alcance del lanzamiento de peso. \\ Biomechanics analysis of the influence in the range of the shot-put with increasing release angle.
}

\author{
Daniel Rojano Ortega \\ Francisco José Berral de la Rosa \\ Universidad Pablo de Olavide, Sevilla
}

\section{Resumen}

El movimiento realizado por el peso en el aire es un movimiento parabólico que, de acuerdo con las leyes físicas, depende de la velocidad de salida, del ángulo de salida y de la altura inicial. La velocidad de salida es más importante que los otros dos factores. Los ángulos óptimos de salida de los lanzadores profesionales son muy bajos. El motivo es que el cuerpo humano está condicionado para ejercer mayor fuerza con ángulos de salida bajos. El objetivo de este trabajo es el de comprobar si, con un entrenamiento adecuado, es posible condicionar a los atletas para ejercer mayor fuerza con ángulos de salida mayores, mejorando así considerablemente el alcance total. Se ha llevado a cabo una investigación con 12 atletas no experimentados. En el pre-test lanzaron el mismo balón medicinal 30 veces con un rango de ángulos de salida de $25^{\circ}$ a $45^{\circ}$. Después de un periodo de entrenamiento de cuatro semanas se llevó a cabo un post-test, similar al inicial. Se realizó un análisis de vídeo en dos dimensiones del mejor lanzamiento de cada sujeto. Se encontraron diferencias significativas en los valores medios de la velocidad de salida, del ángulo de salida y del alcance total, entre el pre-test y el posttest. Las diferencias significativas en la media del ángulo de salida con el que los lanzadores consiguieron sus mejores marcas muestran que el periodo de entrenamiento hizo que los lanzadores desarrollasen más fuerza con ángulos de salida mayores.

Palabras clave: lanzamiento de peso; velocidad de salida; altura inicial; ángulo de salida; distancia de vuelo; ángulo óptimo.

\begin{abstract}
The movement made by the shot in the air is a parabolic movement which, according to physical laws, depends on release speed, release angle and initial height. Release speed is more important than the other two factors. Optimum release angles of professional throwers are very low. The reason for this is that human body is conditioned to exert more force with low release angles. The objective of this work is to asses if, with an appropriate training, it is possible to condition the athletes to exert more force at bigger release angles, improving considerably the range of the shot put. A research has been carried out with 12 inexperienced throwers. In the pre-test they threw the same medicinal ball 30 times over a range of release angles from $25^{\circ}$ to $45^{\circ}$. After a period of four weeks of training, focused on increasing progressively the release angle, a post-test, similar to the initial one, was carried out. All of the throws were filmed and a 2-D video analysis with the best throw of each thrower in both tests was made. There were significant differences in the average values of release speed, release angle and total range between the pre-test and the post-test. The significant differences in the average release angle with which the throwers obtained their best range show that the period of training made the throwers develop more force at bigger release angles.
\end{abstract}

Key words: shot-put; release speed; initial height; release angle; flight distance; optimal angle. 


\section{Introducción}

$\mathrm{E}$ l objetivo del lanzamiento de peso es el de propulsar una bola, llamada peso, de $4 \mathrm{~kg}$ de masa (en el caso de las mujeres) y de 7,26 kg de masa (en el caso de los hombres), a través del aire a una distancia máxima. El lanzamiento se realiza desde un círculo de 2,135 metros de diámetro con un tope o contenedor de $10 \mathrm{~cm}$ de altura en el frente. El peso debe caer dentro de un sector circular de 40 grados situado enfrente del círculo.

Las dos técnicas de lanzamiento más usuales son la técnica dorsal y la rotacional, que difieren en los movimientos preliminares pero no en la fase de liberación (Linthorne, 2001).

Desde un punto de vista físico, el alcance total (R) de un lanzamiento de peso es la suma de dos distancias (Figura 1): la distancia inicial (distancia horizontal a la que se produce el lanzamiento medida desde el borde interno del contenedor, $\left.\mathrm{R}_{0}\right)$ y la distancia de vuelo $\left(\mathrm{R}_{\mathrm{v}}\right)$ (Hay, 1993; Grande et al., 1999).

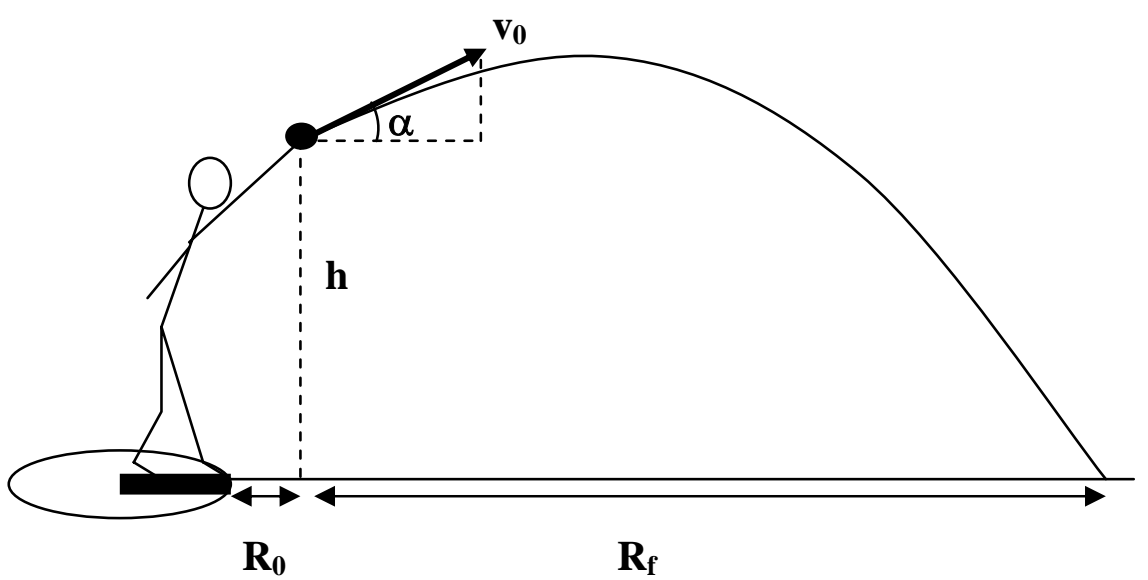

Figura 1. El alcance total es la suma de la distancia inicial $\left(\mathrm{R}_{0}\right)$ y la distancia de vuelo $\left(\mathrm{R}_{\mathrm{v}}\right)$.

En realidad, la distancia oficial es un poco más pequeña porque el peso no impacta en el suelo en un único punto, sino que deja sobre él una marca circular. La distancia oficial es medida desde el borde interno del contenedor hasta la parte de dicha marca circular más próxima al mismo, por lo que la distancia oficial será la suma de las dos distancias anteriores menos el radio de la marca circular que el peso deja al caer. Este valor es denominado por Linthorne (2001), “landing distance” y depende, básicamente, de la firmeza de la superficie, no siendo afectado por la técnica del lanzamiento. Además, la contribución de dicha distancia a la distancia oficial es muy pequeña, por lo que no la tendremos en cuenta en nuestro estudio.

La distancia inicial, que es previa a la liberación del peso, está determinada por la posición final del lanzador y por el ángulo del brazo ejecutor con la horizontal. Esta distancia puede ser positiva o negativa, pero normalmente es muy pequeña. La distancia inicial medida en el Campeonato del Mundo de 1987 fue de $17 \mathrm{~cm}$ de media en los hombres y de $11 \mathrm{~cm}$ de media en las mujeres (Grande et al., 1999). Comparando estos valores con los de la distancia de vuelo parece lógico pensar que una mejora de esta última es la clave para ganar.

La distancia de vuelo depende casi exclusivamente de las condiciones iniciales: velocidad de salida $\left(v_{0}\right)$, ángulo de salida $(\alpha)$ y altura inicial (h), pero también de otros factores derivados del movimiento del peso en el aire (Hochmuth, 1973; Zatsiorsky, Lanka y Shalmanov, 1981, Hay, 1993; Wada, Nishimura, Ogawa, Koyama y Shi, 1994; Bravo, Campos, Durán y Martínez, 2000; Hubbard, 2000; Grande, Meana, López, Tomé y Aguado, 2002). 
La resistencia del aire realiza perturbaciones muy pequeñas a los efectos de la gravedad, por lo que puede ser despreciada (Lichtenberg y Wills, 1978; Hubbard, 1988; de Mestre, 1990). De forma similar, el viento (Lichtenberg y Wills, 1978), la rotación del peso (De Mestre, 1990) y las variaciones en la aceleración debidas a la altitud (Lichtenberg y Wills, 1978), tienen una influencia pequeña en la trayectoria del peso. Por estos motivos, podemos considerar que la distancia de vuelo depende únicamente de las condiciones iniciales.

La expresión matemática para la distancia de vuelo de un proyectil en vuelo libre es la siguiente:

$$
R_{v}=v_{o} \cdot \cos \alpha \cdot \frac{v_{o} \cdot \sin \alpha+\sqrt{\left(v_{o} \cdot \sin \alpha\right)^{2}+2 \cdot g \cdot h}}{g}
$$

El objetivo de un lanzador de peso es el de conseguir las óptimas condiciones iniciales que maximicen la distancia de vuelo. De los tres factores de los que ésta depende, la velocidad de salida tiene el efecto más importante (Hochmuth, 1973; Zatsiorsky et al., 1981, Hay, 1993; Y. Ikegami, Sakurai, Okamoto, Hashimoto y H. Ikegami, 1994; Luhtanen, Korkiakoski y Viitasalo, 1994; Bravo et al., 2000; Hubbard, 2000; Grande et al., 2002), aumentando la distancia de vuelo al aumentar la misma.

Es posible calcular el efecto sobre la distancia de vuelo teórica, obtenida mediante la fórmula anterior, de un incremento similar de los tres factores cinemáticos en un lanzamiento del lanzador español Manuel Martínez (Tabla 1). Comprobamos así que el ángulo de salida y la altura inicial tienen una influencia del mismo orden y mucho menor que la velocidad de salida.

Tabla 1. Efecto sobre la distancia de vuelo de un incremento similar en la velocidad de salida, el ángulo de salida y la altura inicial (adaptada de Grande et al., 1999).

\begin{tabular}{|lcccc|}
\hline VARIABLE & Valores & $\begin{array}{c}\text { Aumento del } \\
\mathbf{5 \%} \mathbf{~ e n ~ h}\end{array}$ & $\begin{array}{c}\text { Aumento del } \\
\mathbf{5 \%} \text { en } \mathbf{~ V}_{\mathbf{0}}\end{array}$ & $\begin{array}{c}\text { Aumento del } \\
\mathbf{5 \%} \mathbf{~ e n} \boldsymbol{\alpha}\end{array}$ \\
\hline Velocidad de salida $\left(v_{0}\right)$ & $13,21 \mathrm{~m} / \mathrm{s}$ & $13,21 \mathrm{~m} / \mathrm{s}$ & $13,87 \mathrm{~m} / \mathrm{s}$ & $13,21 \mathrm{~m} / \mathrm{s}$ \\
\hline Ángulo de salida $(\alpha)$ & $35,9^{\circ}$ & $35,9^{\circ}$ & $35,9^{\circ}$ & $37,69^{\circ}$ \\
\hline Altura inicial $(\mathrm{h})$ & $2,09 \mathrm{~m}$ & $2,19 \mathrm{~m}$ & $2,09 \mathrm{~m}$ & $2,09 \mathrm{~m}$ \\
\hline Distancia de vuelo $\left(R_{v}\right)$ & $19,39 \mathrm{~m}$ & $19,52 \mathrm{~m}$ & $21,17 \mathrm{~m}$ & $19,59 \mathrm{~m}$ \\
\hline Aumento & ------ & $0,13 \mathrm{~m}$ & $1,78 \mathrm{~m}$ & $0,20 \mathrm{~m}$ \\
\hline
\end{tabular}

Un aumento de la altura inicial conlleva siempre un aumento de dicha distancia de vuelo teórica. Con el ángulo de salida ocurre lo mismo hasta un valor óptimo, por encima del cual la distancia disminuye en igualdad de condiciones. Sin embargo, la mayoría de los lanzadores tienen ángulos de salida que están muy por debajo de dichos ángulos óptimos, por lo que también sería conveniente aumentar éste.

La altura inicial está determinada por la posición final del lanzador y por el ángulo que forma el brazo ejecutor con la horizontal. La fase final del lanzamiento acaba con un estiramiento de 
las piernas y una elevación del tronco, de manera que la posición final del lanzador estará determinada por su altura. Esto quiere decir que la altura inicial de un lanzador experimentado sólo podrá verse incrementada por un aumento del ángulo que forma el brazo ejecutor con la horizontal, que es el mismo que el ángulo de salida del peso.

En definitiva, si un aumento del ángulo de salida no va en detrimento de la velocidad de liberación, aumentando el mismo aumentaremos las marcas de los lanzadores.

Muchos autores han estudiado la influencia del ángulo de salida en la distancia de vuelo de un lanzamiento de peso. Lichtenberg y Wills (1978) negaron cualquier dependencia posible entre el ángulo de salida y la velocidad de salida, considerando que los tres factores cinemáticos antes mencionados eran completamente independientes.

El ángulo óptimo de salida sería entonces calculado aplicando a la ecuación de la distancia de vuelo la condición de máximo $\frac{d R_{v}}{d \alpha}=0$, obteniendo (Lichtenberg y Wills, 1978; De Mestre, 1990; Hubbard, 2000):

$$
\alpha_{m}=\operatorname{arctg}\left[\frac{v_{0}}{\sqrt{v_{0}^{2}+2 g h}}\right]
$$

Si los lanzamientos fueran hechos desde el suelo, el ángulo óptimo que maximizaría la distancia de vuelo sería $45^{\circ}$. Cuando el lanzamiento no es realizado desde el suelo, el ángulo óptimo dependerá de la altura inicial y de la velocidad de salida.

Es muy útil representar la distancia de vuelo en función del ángulo de salida para diferentes valores de la velocidad de salida y para un valor fijo de la altura inicial (Figura 2). Con estas curvas es fácil observar el efecto que una desviación del ángulo óptimo de salida tendría sobre la distancia de vuelo.

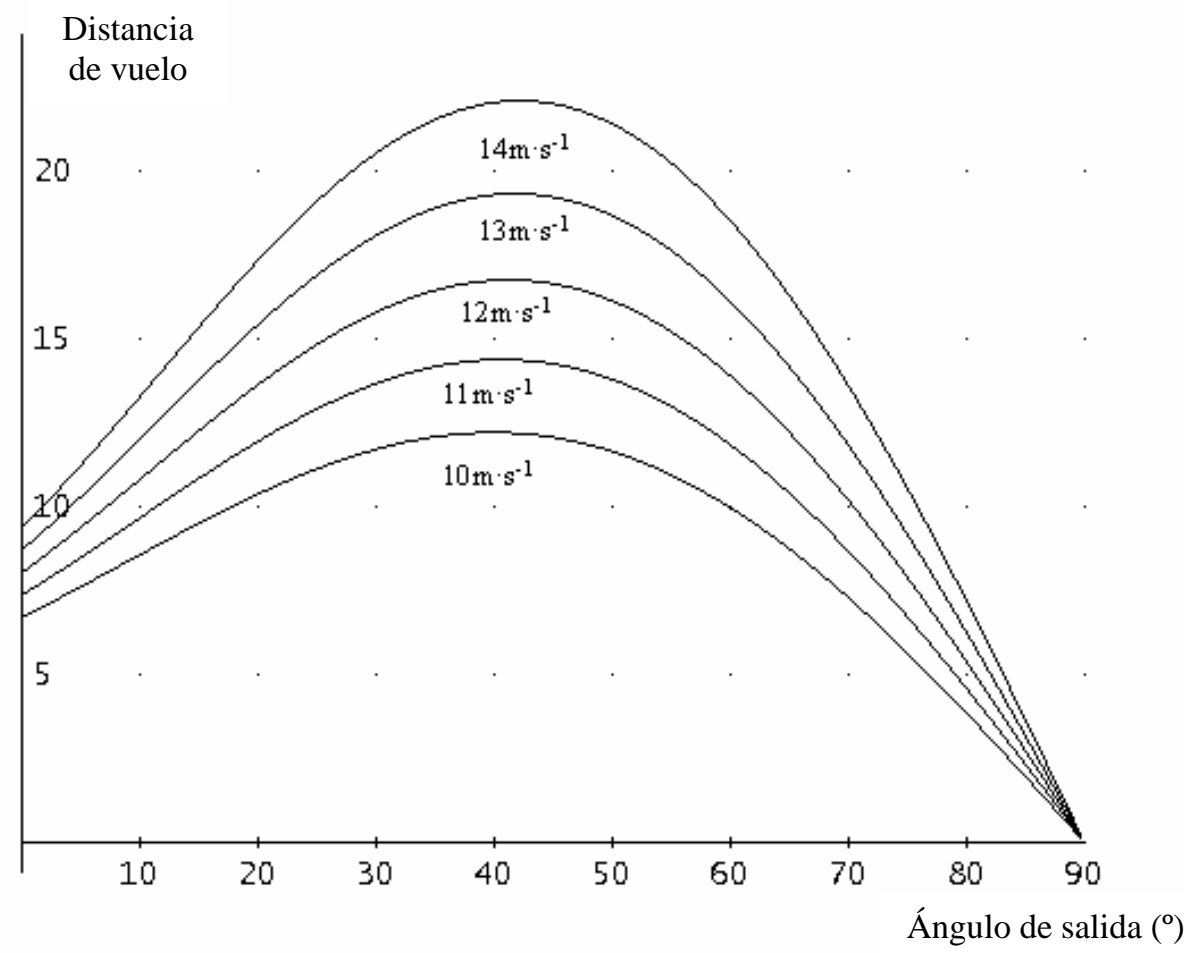

Figura 2. Representación de la distancia de vuelo en función del ángulo de salida para distintos valores de la velocidad de salida y un valor fijo de 2,2 m para la altura inicial. 
Rojano, D.; Berral, F.J. Análisis biomecánico del efecto de un aumento del ángulo de salida en el alcance del lanzamiento de peso. (2009). Revista Internacional de Ciencias del Deporte. 14(5), 94-106.

http://www.cafyd.com/REVISTA/01408.pdf

Si hacemos lo mismo cambiando la altura inicial, podemos concluir que con velocidades de salida mayores que $13 \mathrm{~m} / \mathrm{s}$ y alturas iniciales menores que 2,4 m (valores representativos de un campeonato del mundo), el ángulo óptimo de salida es siempre mayor que 41,5².

Sin embargo, la mayoría de los ángulos de salida en los campeonatos del mundo son mucho menores que los predichos por Lichtenberg y Wills, e incluso a veces menores que $30^{\circ}$ (Susanka y Stepanek, 1988). La consideración de Lichtenberg y Wills (1978) de que los tres factores cinemáticos de los que depende la distancia de vuelo son factores independientes debe ser, por tanto, errónea.

Los tres medallistas del Campeonato del Mundo de 1991 en Tokio, Gunthoer, Andersen y Nielsen tuvieron ángulos de salida en sus mejores lanzamientos de $36,1^{\circ}, 37,0^{\circ}$ y 37,9 ${ }^{\circ}$ respectivamente (Bravo et al., 2000). El lanzador español Manuel Martínez ganó el Campeonato de España de 1996 con un lanzamiento con un ángulo de salida de 35,9² (Bravo et al., 2000).

El motivo de que estos ángulos sean tan bajos es que el esfuerzo necesitado para proyectar horizontalmente el peso parece ser mucho menor que el necesitado para proyectarlo verticalmente (Zatsiorsky et al., 1981). Si el ángulo de salida aumenta, el efecto de la gravedad aumenta también, y no es posible obtener la misma velocidad de salida que cuando los ángulos son bajos (Hay, 1973). Según Linthorne (2001) este motivo haría disminuir unos $2^{\circ}$ los ángulos óptimos predichos por Lichtenberg y Wills (1978), pero estos valores aún estarían muy por encima de los conseguidos por los lanzadores de primer nivel.

Algunos autores (McCoy et al., 1984; McWatt, 1982) argumentan además que el cuerpo humano es capaz de producir más fuerza cuando el peso es lanzado con ángulos de salida bajos.

Dos investigaciones usando una bola de 1,14 kg de masa (Red y Zogaib, 1977) y una jabalina (Viitasalo y Korjus, 1988) determinaron que la velocidad de salida decrecía linealmente con el ángulo de salida. Maheras (1995) hizo un estudio experimental con cinco lanzadores que lanzaban con un rango de ángulos de salida desde $16^{\circ}$ hasta $54^{\circ}$, encontrando que la velocidad de salida decrecía linealmente al aumentar el ángulo de salida. Hubbard et al. (2001) realizaron una investigación con dos lanzadores de peso experimentados estableciendo que la velocidad de salida decrecía al aumentar el ángulo de salida a razón de 1,7 (m/s)/rad.

Red y Zogaib (1977) propusieron un método para calcular el ángulo de salida óptimo real en el lanzamiento de jabalina. Ellos observaron un decrecimiento lineal de la velocidad de salida al aumentar el ángulo de salida en tres lanzadores. Para obtener el ángulo óptimo de salida real para cada lanzador combinaron la ecuación empírica que relacionaba la velocidad de salida y el ángulo de salida de sus lanzadores con las ecuaciones que describen la trayectoria de la jabalina en vuelo libre. El ángulo así obtenido coincidía bastante bien con el ángulo de salida correspondiente a los mejores lanzamientos pero, para ser aún más preciso, se debería haber incluido también la relación entre la altura inicial y el ángulo de salida.

Más tarde, Linthorne (2001) usó los datos de Maheras para comprobar la exactitud del método de Red y Zogaib (1977) al calcular el ángulo óptimo propio de cada lanzador en el lanzamiento de peso.

Con los datos de Maheras, Linthorne (2001) obtuvo expresiones algebraicas para la velocidad de salida y la altura inicial en función del ángulo de salida, usando el método de los mínimos cuadrados. Estas expresiones fueron sustituidas en la ecuación de la distancia de vuelo, obteniendo una relación directa entre la distancia de vuelo y el ángulo de salida. En esta relación asumió que la trayectoria del peso en la fase de liberación era recta y de una longitud 
aproximada de 1,65 m (Susanka y Stepanek, 1988), y también fijó el trabajo realizado por el atleta durante esta fase.

Para calcular dicho trabajo, Linthorne (2001) consideró dos modelos diferentes: en el primero toma la fuerza como constante e independiente del ángulo de salida y en el segundo considera que la fuerza decrece linealmente al aumentar el ángulo de salida.

McCoy et al. (1984) sugirieron que los atletas son capaces de producir más fuerza con ángulos de salida bajos. Un lanzamiento con un ángulo de salida bajo simula la posición del tronco y del brazo durante un press de banca y los lanzadores están mejor condicionados para la realización de este ejercicio que para un press de hombros, ejercicio que simularía un lanzamiento con un ángulo de salida alto. McWatt (1982) estaba de acuerdo con ellos, basando su opinión en el hecho de que individuos no entrenados mostraban mayor fuerza isométrica de hombros cuando ejercían la fuerza con ángulos bajos.

Por estas razones, Linthorne (2001) sugirió que el segundo modelo era más apropiado. Los valores que él obtuvo con este segundo modelo para el ángulo óptimo de salida coincidían bastante bien con los ángulos de salida de los mejores lanzamientos de los atletas analizados por Maheras y estos ángulos debían estar cerca de los ángulos óptimos reales de salida puesto que se trataba de lanzadores de nivel mundial.

No obstante, en nuestra opinión, esta asunción de Linthorne (2001) está basada en hipótesis confirmadas por estudios transversales con atletas o sujetos no entrenados que están probablemente condicionados para ejercer mayor fuerza con ángulos bajos. Sin embargo, no realiza un estudio longitudinal para saber si es posible modificar la dirección en la que se ejerce mayor fuerza.

Además, Linthorne encontró una constante que caracterizaba el decrecimiento en la fuerza realizada por cada atleta al aumentar el ángulo de salida. Esta constante era específica para cada atleta, lo que nos lleva aún más a pensar en la posibilidad de modificar la dirección en la que los atletas ejercen la mayor fuerza.

El objetivo de este trabajo es entonces verificar si, con un entrenamiento apropiado, es posible condicionar a los atletas para ejercer mayor fuerza con ángulos de salida mayores o para ejercer la misma fuerza en un rango mayor de ángulos de salida, de forma que el ángulo de salida óptimo sea de unos 39,50 (valor obtenido por Linthorne (2001) al considerar la fuerza realizada como constante e independiente de la dirección).

\section{Material y métodos}

Se ha formado un grupo de doce estudiantes de la Universidad Pablo de Olavide de Sevilla, con edades comprendidas entre 20 y 25 años. Ninguno de ellos era lanzador experimentado, pero todos tenían nociones básicas de la técnica de deslizamiento. Se ha llevado a cabo una investigación sobre los valores del ángulo de salida con el que los lanzadores conseguían sus mejores marcas. Todos los sujetos dieron su consentimiento informado, de acuerdo con la Declaración de Helsinki.

Se realizó un test inicial consistente en un pequeño calentamiento descrito más adelante y 30 lanzamientos simulando la técnica de deslizamiento, pero sin realizar el mismo o realizando un deslizamiento muy pequeño con objeto de permanecer en el plano de calibración. Los lanzamientos se realizaron con un balón medicinal de $2 \mathrm{~kg}$ de masa y $20 \mathrm{~cm}$ de diámetro. Hubo dos sesiones distintas para así asegurar que los lanzamientos fueran realizados sin fatiga. Se 
Rojano, D.; Berral, F.J. Análisis biomecánico del efecto de un aumento del ángulo de salida en el alcance del lanzamiento de peso. (2009). Revista Internacional de Ciencias del Deporte. 14(5), 94-106.

informó a los sujetos de que los lanzamientos debían ser realizados con máximo esfuerzo y lanzando primero con un ángulo bajo e intentando incrementar un poco dicho ángulo cada 5 lanzamientos.

El objetivo era cubrir un rango de ángulos de salida de $25^{\circ}$ a $45^{\circ}$. Se buscó que en cada intervalo de $2^{\circ}$ siempre hubiese algún lanzamiento. Para aquellos sujetos en los que no se cumplía este requisito se realizó una tercera sesión complementaria de lanzamientos orientada a obtener los correspondientes ángulos de salida.

Al final, después de un periodo de entrenamiento de cuatro semanas, se llevó a cabo un nuevo test, similar al inicial. Durante este periodo los lanzadores siguieron una serie de ejercicios destinados incrementar el ángulo de salida con el que conseguían su mejor alcance.

Se filmaron todos los lanzamientos de cada test. Los lanzamientos se grabaron con una cámara de alta velocidad (JAI - CV - A, a 100 fotogramas por segundo), que se fijó perpendicularmente al plano (XY) del círculo de lanzamientos donde el movimiento debía ocurrir (plano de calibración). La cámara se situó a 10 m de distancia de dicho plano. En cada test y al final de cada semana de entrenamiento se realizó un análisis en dos dimensiones (2D) del mejor lanzamiento de cada sujeto. La calibración de la cámara se realizó con un objeto de dimensiones conocidas situado en el plano de calibración.

Los vídeos se trataron con un programa de edición de vídeo con el que seleccionamos los dos primeros fotogramas en los que el atleta acababa de lanzar el balón. Con el programa Corel Draw 12 superpusimos dichos fotogramas y medimos la altura inicial y el espacio horizontal y vertical recorrido por el balón entre los dos fotogramas. Con estos datos y las ecuaciones cinemáticas necesarias calculamos la velocidad de salida y el ángulo de salida. El alcance medido fue la proyección del alcance real sobre el plano de calibración.

En el transcurso de un lanzamiento real, el movimiento no permanece totalmente en el plano de calibración y, dado que nuestro análisis es en dos dimensiones, habrá errores como consecuencia del factor de escala erróneo. Sin embargo, nuestros cálculos están basados en los dos primeros fotogramas, los lanzamientos fueron hechos desde una posición casi estática y los lanzamientos que en su caída se habían alejado más de 1m del plano de calibración fueron descartados. Por estas razones, con los tiempos de vuelo con los que tratamos, la posible separación del plano de calibración en los dos primeros fotogramas sería únicamente de unos centímetros y, dado que la cámara estaba situada a $10 \mathrm{~m}$ de distancia del plano de calibración, los errores derivados del factor de escala son despreciables.

Además, como nuestro estudio es en dos dimensiones, sería posible que el lanzamiento con mayor alcance real no tuviese la mayor proyección sobre el plano de calibración (como consecuencia de la pérdida de la componente Z). Podríamos haber considerado así como mejores lanzamientos algunos que en realidad no lo habrían sido. Este es el motivo por el cual antes de comenzar este estudio realizamos un análisis de 20 lanzamientos realizados en las mismas condiciones que en nuestra investigación e hicimos una correlación entre el alcance real y la proyección del mismo sobre el plano de calibración, obteniendo un coeficiente de correlación de 0,974 con un nivel de confianza de 0,99 , lo que significa que podemos asumir, sin prácticamente riesgo a equivocarnos, que los componentes de la velocidad en el plano de calibración son los que determinan qué lanzamientos tienen mayor alcance.

Las sesiones de entrenamientos estuvieron basadas en indicaciones obtenidas por la Real Federación Española de Atletismo. Se realizaron tres sesiones de 1,30 horas de entrenamiento cada semana. 
Al comienzo de las mismas se llevó a cabo un calentamiento de unos 15 minutos consistente en una carrera suave de 5 minutos seguida de estiramientos y de ejercicios de movilidad articular. Después se realizaban algunos ejercicios para trabajar los músculos abdominales, lumbares y oblicuos para comenzar a continuación a realizar ejercicios específicos de lanzamientos. Este es el mismo calentamiento que se realizó en el pre-test y en el post-test.

Los ejercicios de lanzamientos estaban encaminados a aumentar los ángulos de salida con los que los lanzadores conseguían mejores marcas. Consistían en lanzamientos de distintos tipos realizados a una mano o a dos, destinados básicamente a aumentar el ángulo de salida final.

Las sesiones de lanzamientos se organizaron de la siguiente manera:

o 30 lanzamientos con balones medicinales de 1, 2 y $3 \mathrm{~kg}$ de masa (10 lanzamientos con cada peso) con una intensidad comprendida aproximadamente entre el $60 \%$ y el $80 \%$ de la capacidad total del lanzador. De cada diez lanzamientos se realizaban 5 con un brazo y 5 con el otro, aunque a veces, para dar mayor variedad a los entrenamientos se realizaban algunos a dos manos.

o 15 lanzamientos con un balón medicinal de 2 kg realizados a la máxima intensidad, con el brazo dominante y descansando el tiempo necesario para evitar la fatiga.

o 15 lanzamientos de baja intensidad realizados con balones medicinales de 1 ó $2 \mathrm{~kg}$ en función del cansancio del lanzador y alternando el brazo ejecutor.

Los lanzamientos realizados durante los entrenamientos se ejecutaban de la misma manera que en el pre-test y en el post-test.

El problema real de las sesiones de entrenamiento consistía en cómo asegurar que los lanzamientos se estaban realizando con ángulos de salida mayores que los habituales. Para elaborar nuestros lanzamientos dimos preferencia a la operatividad del entrenamiento diseñando los ejercicios para un lanzador cuyos parámetros fuesen los valores medios de nuestros lanzadores. En realidad estos ejercicios deberían estar personalizados para cada uno, por lo que asumíamos que estábamos cometiendo errores bastante difíciles de cuantificar pero, en cualquier caso, muy pequeños.

Para el diseño de los lanzamientos de la primera semana tuvimos en cuenta el mejor lanzamiento de cada atleta realizado durante el pre-test. En dichos lanzamientos, además de la velocidad de salida, el ángulo de salida y el alcance total, medimos muchos otros parámetros, entre los que nos interesaron especialmente la altura del hombro en el momento del lanzamiento, la distancia entre el hombro y el centro del balón en el momento justo de la liberación del mismo y la distancia inicial del lanzamiento.

Situamos dos soportes verticales con dos barras horizontales graduables en altura delante del círculo de lanzamientos y a una distancia horizontal del borde interno del contendor de $1 \mathrm{~m}$. El objetivo era que el balón pasara entre las dos barras horizontales y, para calcular la altura a la que debíamos situar las barras horizontales, impusimos que los ángulos de salida estuviesen comprendidos entre $40^{\circ}$ y $45^{\circ}$, para que incluso los lanzadores cuya altura inicial fuese mayor que la media estuviesen incrementando su ángulo de salida. 
Las alturas correspondientes las calculamos basándonos en la figura 3:

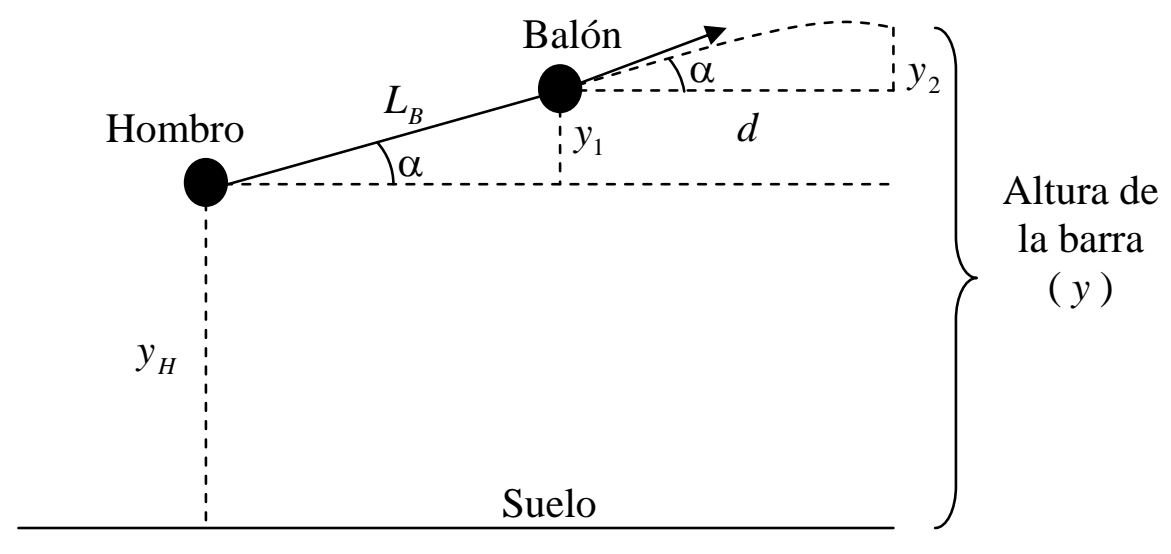

Figura 3. Representación del hombro del lanzador y del balón en el momento de la liberación.

Utilizando trigonometría básica y las ecuaciones cinemáticas que gobiernan el movimiento parabólico llegamos a la siguiente ecuación para la altura de las barras:

$$
y=y_{H}+y_{1}+y_{2}=y_{H}+L_{B} \cdot \operatorname{sen} \alpha+v \cdot \operatorname{sen} \alpha \cdot\left(\frac{d}{v \cdot \cos \alpha}\right)-\frac{1}{2} \cdot g \cdot\left(\frac{d}{v \cdot \cos \alpha}\right)^{2}
$$

Siendo:

$y$ : altura de la barra.

$y_{H}$ : altura media del hombro.

$L_{B}$ : longitud media entre el hombro y el centro del balón en la salida.

$\alpha$ : ángulo de salida.

$g$ : aceleración de la gravedad.

$d$ : valor medio de la distancia horizontal entre el centro del balón y la barra en la salida.

Los valores de d los obtenemos restando a $1 \mathrm{~m}$ (distancia horizontal a la que se sitúa la barra respecto al borde interno del contenedor) la distancia inicial del lanzamiento.

Así, sustituyendo los ángulos por $40^{\circ}$ y $45^{\circ}$ y el resto de parámetros por los valores obtenidos en el pre-test obtuvimos las alturas a las que debíamos situar las barras. Teniendo en cuenta que el balón medicinal tenía un radio de $10 \mathrm{~cm}$, fue necesario sumar $10 \mathrm{~cm}$ a la más alta y resta $10 \mathrm{~cm}$ a la más baja.

En el último entrenamiento de cada semana filmamos los 15 lanzamientos realizados a máximo nivel e hicimos lo mismo que en el pre-test para así calcular las alturas de las barras en la semana siguiente. 
Rojano, D.; Berral, F.J. Análisis biomecánico del efecto de un aumento del ángulo de salida en el alcance del lanzamiento de peso. (2009). Revista Internacional de Ciencias del Deporte. 14(5), 94-106.

\section{Resultados}

Con los valores de los parámetros cinemáticos del lanzamiento de mayor alcance de cada sujeto en el pre-test, hicimos una estadística básica para conocer los valores medios del ángulo de salida, de la velocidad de salida y del alcance total (Tabla 2). Los doce lanzamientos fueron realizados con una velocidad de salida media de 10,75 m/s, un ángulo de salida medio de $37,34^{\circ}$ y tuvieron un alcance medio de $13,99 \mathrm{~m}$.

Tabla 2. Estadística básica de la media de los tres mejores lanzamientos de cada sujeto en el pre-test.

\begin{tabular}{|l|c|c|c|c|c|}
\hline & $\mathrm{N}$ & Mínimo & Máximo & Media & D. Estándar \\
\hline Velocidad de salida (m/s) & 12 & 9,76 & 12,16 & 10,7517 &, 78550 \\
\hline Ángulo de salida $\left(^{\circ}\right)$ & 12 & 31,64 & 42,84 & 37,3492 & 3,70578 \\
\hline Alcance total (m) & 12 & 12,62 & 15,94 & 13,9900 & 1,18546 \\
\hline Valid N (listwise) & 12 & & & & \\
\hline
\end{tabular}

En el post-test, después de cuatro semanas de entrenamiento, los doce lanzamientos tuvieron una velocidad de salida media de $11,44 \mathrm{~m} / \mathrm{s}$, un ángulo de salida medio de $40,96^{\circ}$ y un alcance medio de 15,22 m (Tabla 3).

Tabla 3. Estadística básica de la media de los tres mejores lanzamientos de cada sujeto en el post-test.

\begin{tabular}{|l|c|c|c|c|c|}
\hline & $\mathrm{N}$ & Mínimo & Máximo & Media & D. Estándar \\
\hline Velocidad de salida (m/s) & 12 & 10,46 & 12,15 & 11,4358 &, 58277 \\
\hline Ángulo de salida ( $\left.{ }^{\circ}\right)$ & 12 & 36,17 & 44,92 & 40,9558 & 2,72054 \\
\hline Alcance total (m) & 12 & 13,86 & 16,90 & 15,2217 & 1,15607 \\
\hline Valid N (listwise) & 12 & & & & \\
\hline
\end{tabular}

Hicimos una prueba t de Student para muestras apareadas para saber si había diferencias significativas en la media de los tres parámetros entre el pre-test y el post-test (Tabla 4). La prueba mostró que con un nivel de confianza del $95 \%$, había diferencias significativas en las medias de los tres parámetros, porque los tres habían aumentado de manera notable.

Tabla 4. Test t-Student para muestras dependientes para comprobar la existencia de diferencias significativas entre el pre-test y el post-test.

\begin{tabular}{|c|c|c|c|c|c|c|c|c|c|}
\hline & & & & red Differer & & & & & \\
\hline & & & & & $\begin{array}{r}95 \% \mathrm{C} \\
\text { Interv } \\
\text { Diffe }\end{array}$ & $\begin{array}{l}\text { idence } \\
\text { of the } \\
\text { nce }\end{array}$ & & & \\
\hline & & Mean & Deviation & Mean & Lower & Upper & $\mathrm{t}$ & df & tailed) \\
\hline $\begin{array}{l}\text { Pair } \\
1\end{array}$ & $\begin{array}{l}\text { Velocidad de salida pre- } \\
\text { test }(\mathrm{m} / \mathrm{s}) \text { - Velocidad de } \\
\text { salida post-test }(\mathrm{m} / \mathrm{s})\end{array}$ &,- 68417 & 40464 & 11681, &,- 94126 &,- 42707 & $-5,857$ & 11 & , 000 \\
\hline $\begin{array}{l}\text { Pair } \\
2\end{array}$ & $\begin{array}{l}\text { Ángulo de salida pre-test } \\
\left({ }^{\circ}\right) \text { - Ángulo de salida post- } \\
\text { test }\left(^{\circ}\right)\end{array}$ & $-3,60667$ & 4,73893 & 1,36801 & $-6,61764$ &,- 59570 & $-2,636$ & 11 & ,023 \\
\hline $\begin{array}{l}\text { Pair } \\
3\end{array}$ & $\begin{array}{l}\text { Alcance total pre-test }(\mathrm{m}) \text { - } \\
\text { Alcance total post-test }(\mathrm{m})\end{array}$ & $-1,23167$ & 51767, & 14944 & $-1,56058$ & -,90276 & $-8,242$ & 11 & ,000 \\
\hline
\end{tabular}




\section{Discusión}

Los resultados obtenidos en la estadística básica del pre-test y del post-test muestran que la media de la velocidad de salida, del ángulo de salida y del alcance total han aumentado considerablemente después de las cuatro semanas de entrenamiento. La velocidad de salida ha aumentado desde $10.75 \mathrm{~m} / \mathrm{s}$ hasta $11.44 \mathrm{~m} / \mathrm{s}$, lo que supone un $6.42 \%$; el ángulo de salida ha aumentado desde $37.35^{\circ}$ hasta $40.96^{\circ}$, lo que supone un $9.66 \%$ y el alcance total ha variado de $13.99 \mathrm{~m}$ a $15.22 \mathrm{~m}$, es decir, ha aumentado un $8.79 \%$.

La existencia de diferencias significativas entre el pre-test y el post-test en los valores medios del alcance total y de la velocidad de salida no constituye una información relevante puesto que los sujetos no eran experimentados y, después de cuatro semanas de entrenamiento, es completamente normal que dichos valores hayan aumentado de forma significativa.

Sin embargo, la existencia de diferencias significativas en el valor medio del ángulo de salida del mejor lanzamiento de cada sujeto significa que el entrenamiento ha hecho que los lanzadores ejerzan mayor fuerza con ángulos de salida mayores, incluso mayores que los $39,5^{\circ}$ predichos por Linthorne (2001) cuando consideraba la fuerza desarrollada independiente de la dirección de lanzamiento.

\section{Conclusiones}

Los resultados obtenidos en la prueba t de Student para los valores medios del ángulo de salida de los lanzamientos con los que los atletas han conseguido sus mejores marcas nos hacen pensar que la dirección en la que cada sujeto es capaz de ejercer mayor fuerza no es algo intrínseco de cada persona, sino que se puede modificar con un entrenamiento adecuado, al contrario de lo que piensan muchos autores.

Al tratarse de lanzadores no experimentados es lógico que los valores medios del alcance total y, por tanto, de la velocidad de salida hayan sido bastante mayores en el post-test que en el pre-test. Sin embargo, no sabemos realmente si ese aumento en el ángulo de salida ha afectado negativamente a la velocidad de salida, no permitiendo un mayor aumento de ésta. Es por esta razón por la que estos resultados no pueden extrapolarse directamente a lanzadores experimentados, ya que la explosividad de los mismos podría decrecer al cambiar la dirección en la que ejercen mayor fuerza, de forma que los valores de la velocidad de salida fueran menores que los obtenidos antes del periodo de entrenamiento y el alcance total también lo fuera.

Por este motivo consideramos que sería interesante llevar a cabo entrenamientos similares y más personalizados con grupos de lanzadores profesionales contrastando sus resultados con los de un grupo de control. Así, si nuestras hipótesis fueran confirmadas, los entrenamientos de los lanzadores deberían hacer mayor hincapié en ejercer la máxima fuerza con ángulos de salida de unos $40^{\circ}$, variando algo este valor en función de las características del lanzador.

\section{Agradecimientos}

Los autores agradecen su colaboración a los atletas Julián Arcos, Luís A. González, Jesús Hormigo, Juan M. Lara, José M. López, Antonio J. Macías, José Madrigal, Carlos Martínez, David Moncada, Cristóbal Montoro, Ismael Moya y Abel Pérez de la Universidad Pablo de Olavide de Sevilla. 
Rojano, D.; Berral, F.J. Análisis biomecánico del efecto de un aumento del ángulo de salida en el alcance del lanzamiento de peso. (2009). Revista Internacional de Ciencias del Deporte. 14(5), 94-106.

\section{Referencias}

Bravo, J.; Campos, J.; Durán, J.; Martínez, J.L., 2000. Lanzamientos, Atletismo III. Madrid: Real Federación Española de Atletismo.

De Mestre, N., 1990. The Mathematics of Projectiles in Sport. Cambridge: Cambridge University Press.

Garfoot, B.P. 1968. Analysis of the trajectory of the shot. Track Technique 32, pp. 1003 1006.

Grande, I.; Burón, C.; López, J.L.; Meana, M.; Tomé, I.; Aguado, X., 1999. El apoyo biomecánico al rendimiento deportivo: Estudio biomecánico del lanzamiento de peso en competición. Su aplicación a la construcción de ejercicios específicos de entrenamiento. Madrid: serie ICd de Investigaciones en Ciencias del Deporte. Ministerio de Educación y Ciencia. Consejo Superior de Deportes. № 22.

Grande, I.; Meana, M.; López, J.L.; Tomé, I.; Aguado, X., 2002. Biomecánica del lanzamiento de peso: Estudio del modelo técnico individual. Archivos de Medicina del Deporte 19, pp. 187 - 195.

Hay, J.G., 1993. The Biomechanics of Sports Techniques. New Jersey: Prentice Hall.

Hochmuth, G., 1973. Biomecánica de los Movimientos Deportivos. Madrid: Editorial Instituto Nacional de Educación Física.

Hubbard, M., 1988. The throwing events in track and field. The Biomechanics of Sport, pp. 213 - 238. CRC Press, Boca Raton.

Hubbard, M., 2000. The flight of sports projectiles. In: Zatsiorsky, V.M. (Ed.), Biomechanics in Sport: Performance Enhancement and Injury Prevention. Blackwell Science, Ltd., Oxford: pp. $381-400$.

Hubbard, M. ; De Mestre, N. ; Scott, J., 2001. Dependence of release variables in the shot put. Journal of Biomechanics 34, pp. $449-456$.

Ikegami, Y.; Sakurai, S.; Okamoto, A.; Hashimoto, I.; Ikegami, H., 1994. Biomechanics of shot put. Journal of Biomechanics 27, pp. 670.

Lichtenberg, D.B., Wills, J.K.G., 1978. Maximizing the range of the shot put. American J ournal of Physics 46, pp. 546 - 549.

Linthorne, N.P., 2001. Optimum release angle in the shot put. Journal of Sports Sciences 19, pp. $359-372$.

Luhtanen, P.; Korkiakoski, V.; Viitasalo, J.T., 1994. Influence of the shot weight on the range, velocity and power in shot put. Journal of Biomechanics 27, pp. 674.

McCoy, R.W., Gregor, R.J., Whiting, W.C., Rich, R.G., Ward, P.E., 1984. Kinematic analysis of elite shot putters. Track Technique 90, pp. 2868-2871.

Maheras, A.V., 1995. The relationship between the angle of release and velocity of release in the shot put, and the application of a theoretical model to estimate the optimum angle of release. Ph. D.Thesis. Universityof Kansas.

McWatt, B., 1982. Angles of release in the shot put. Modern Athlete and Coach 20 (4), pp. 17 - 18.

Red, W. E.; Zogaib, A.J., 1977. Javelin dynamics including body interaction. Journal of Applied Mechanics 44, pp. 496 - 498.

Susanka, P.; Stepanek, J., 1988. Biomechanical analysis of the shot put. In Scientific Report on the Second IAFF World Championship in Athletics, 2nd edn., pp. I/1 - I/77. Monaco: IAFF. 
Rojano, D.; Berral, F.J. Análisis biomecánico del efecto de un aumento del ángulo de salida en el alcance del lanzamiento de peso. (2009). Revista Internacional de Ciencias del Deporte. 14(5), 94-106.

Viitasalo, J.T.; Korjus, T., 1988. On-line measurement of kinematic characteristics for javelin. In: deGroot, G. et al., 1988. Biomechanics XI-B, Free University Press, Amsterdam, pp. 582 - 587.

Wada, Y.; Nishimura, T.; Ogawa, K.; Koyama, Y.; Shi, X.T., 1994. On kinematic analysis for throwing forms of the shot put. Journal of Biomechanics 27: pp. 695.

Zatsiorsky, V.M.; Lanka, G.E.; Shalmanov, A.A., 1981. Biomechanical analysis of shot putting technique. Exercise and Sport Sciences Reviews 9, pp. 353 - 389. 